\title{
Microwave-assisted synthesis of quinoline alkaloids: 4-Methoxy-1-methyl-2-quinolinone and its analogs
}

\author{
Vetrivel Nadaraj, Senniappan Thamarai Selvi,* and Raju Sasi \\ Department of Chemistry (PG \& Research) Kongunadu Arts \& Science College G.N.Mills Post, \\ Coimbatore-641 029, INDIA \\ E-mail: thamaraimohan@yahoo.co.in
}

\begin{abstract}
The microwave-induced synthesis of some quinoline alkaloids of the type 4-methoxy-1-methyl2-quinolinone including folimine is reported. The precursors 4-hydroxy-2-quinolinone derivatives were effectively prepared in a single step from aniline and diethylmalonate using $p$ toluenesulfonic acid as a catalyst and these intermediates were converted to the titled quinoline alkaloids by treatment with dimethyl sulfate, N,N-dimethylformamide and potassium carbonate. The presented synthentic procedure is a convenient, simple and fast alternative for synthesizing 4-methoxy-1-methyl-2-quinolinone and its derivatives.
\end{abstract}

Keywords: Quinolines, microwave irradiation, 4-hydroxy-2-quinolinones, 4-methoxy-1-methyl2-quinolinones

\section{Introduction}

The root stock of quinoline alkaloids is specific to the plant family Rutaceae embodying about hundred and fifty genera with sixteen hundred species. The presence of quinoline nucleus in the framework of various pharmacologically active compounds with antiasthmatic, ${ }^{1}$ antibacterial, ${ }^{2}$ antifungal, ${ }^{3}$ antimalarial, ${ }^{4}$ anti-viral, ${ }^{5}$ anti-inflammatory ${ }^{6}$ activities continue to promote their synthetic efforts. Among the quinolines, some facile precursors are available which could be transformed into desired quinoline alkaloids using various reactions. ${ }^{7-9}$ For instance, 4-hydroxy2-quinolinone is an important biosynthetic ${ }^{7}$ and synthetic ${ }^{8,9}$ precursor of quinoline alkaloids. Methylated compounds of this type like 4-methoxy-1-methyl-2-quinolinone (1), folimine (2), 4,6-dimethoxy-1-methyl-2-quinolinone and 4,7,8-trimethoxy-1-methyl-2-quinolinone (Scheme 1) are widely distributed in nature. ${ }^{10-13}$ Though these alkaloids are natural products, they are also used as synthetic intermediates for the preparation of other quinoline alkaloids ${ }^{14}$ and polyheterocycles. 
<smiles>[R]c1cc2c(OC)cc(=O)n(C)c2c([R])c1[R]</smiles>

$1 \mathrm{R}^{1}=\mathrm{R}^{2}=\mathrm{R}^{3}=\mathrm{H}$; 4-Methoxy-1-methyl-2-quinolinone

$2 \mathrm{R}^{1}=\mathrm{R}^{2}=\mathrm{H}, \mathrm{R}^{3}=\mathrm{OCH}_{3}$; Folimine

$3 \mathrm{R}^{2}=\mathrm{R}^{3}=\mathrm{H}, \mathrm{R}^{1}=\mathrm{OCH}_{3} ; 4,6$-Dimethoxy-1-methyl-2-quinolinone

$4 \mathrm{R}^{1}=\mathrm{H}, \mathrm{R}^{2}=\mathrm{R}^{3}=\mathrm{OCH}_{3} ; 4,7,8$-Trimethoxy-1-methyl-2-quinolinone

\section{Scheme 1}

The classical synthetic protocols for the above quinoline intermediates and natural products suffer from some disadvantages such as low yield, ${ }^{15}$ lack of easy availability/ preparation of the reagent, ${ }^{16-18}$ prolonged reaction time, multiple steps, requirement of excess of reagents/catalysts, need for special apparatus and harsh reaction conditions. ${ }^{16,17}$ In this connection and by knowing the advantages of microwave reactions, ${ }^{19-21}$ we felt that the synthesis of the above intermediates and quinoline alkaloids using microwave irradiation in an unaltered domestic microwave oven would make the task much easier than the thermal synthesis. Hence we wish to report a convenient and efficient synthesis of 4-hydroxy-2-quinolinone and 4methoxy-1-methyl-2-quinolinone derivatives under microwave irradiation.

\section{Results and Discussion}

In an initial study, a mixture of aniline and diethylmalonate (2:1 molar ratio) was taken in a beaker (without adding any support, i.e., neat conditions), and irradiated under microwaves. This type of reaction is expected to be the most economical method since neither catalyst nor solvent is used. We failed to obtain 4-hydroxy-2-quinolinone (5a); but the $N, N$ '-diphenyl malonamide (6a) was formed in $20 \mathrm{~min}$ (90\% yield) (Scheme 2). This result agreed with the report given by Lange et $\mathrm{al}^{22}$ in their synthesis of 3-phenyl substituted 4-hydroxy-2-quinolinones. By contrast, in the presence of a catalytic amount of $p$-toluenesulfonic acid, the quinolinone 5a was isolated in $89 \%$ yield in 6 min under the same reaction conditions (Scheme 2). IR spectrum of 5a exhibited the absorption bands at $1660,1606 \mathrm{~cm}^{-1}$ ascribable to carbonyl groups and a broad $\mathrm{NH}$ absorption in the region 3400-2900 $\mathrm{cm}^{-1}$. The ${ }^{1} \mathrm{H}-\mathrm{NMR}$ spectrum represented a singlet at $\delta 5.80$ for the $\mathrm{C}_{3}$-proton, a multiplet in the region $\delta$ 7.05-7.65 for aromatic protons and a doublet at $\delta 7.70$ for $\mathrm{C}_{5}$-proton. The singlet at $\delta 10.35$ is accounted for $\mathrm{NH}$ proton and a broad singlet at $\delta$ 10.55 is due to the $-\mathrm{OH}$ group. 
Table 1. Effect of solid support for the synthesis of $\mathbf{5 a}$ under microwave irradiation (Power $=$ 320W)

\begin{tabular}{lcc}
\hline Support & Time $(\min )$ & Yield (\%) \\
\hline No & 20 & - \\
Silica gel & 12 & 55 \\
Acidic alumina & 12 & 46 \\
$\mathrm{~K}_{10}$ clay & 12 & 41 \\
$p$-Toluenesulfonic acid & 6 & 89 \\
\hline
\end{tabular}

Different solid supports, including silica gel, acidic alumina and montmorillonite $\left(\mathrm{K}_{10}\right.$ clay) were also checked to define the most effective catalyst (Table 1). From these results, it is obvious that $p$-toluenesulfonic acid is the most adaptable and simplest catalyst for the synthesis of 5a, as work-up is simply reduced to treatment with ice-cold water. Thus, we have extended these conditions to the synthesis of various 4-hydroxy-2-quinolinones (5a-g) with high yields (Scheme 3; Table 2). It was also attempted to convert N,N'-diphenyl malonamide (6a) to 4hydroxy-2-quinolinone (5a) by microwave irradiation, in which the reaction was (Scheme 2) completed with in $3 \mathrm{~min}$, in presence of the catalyst $p$-toluenesulfonic acid.

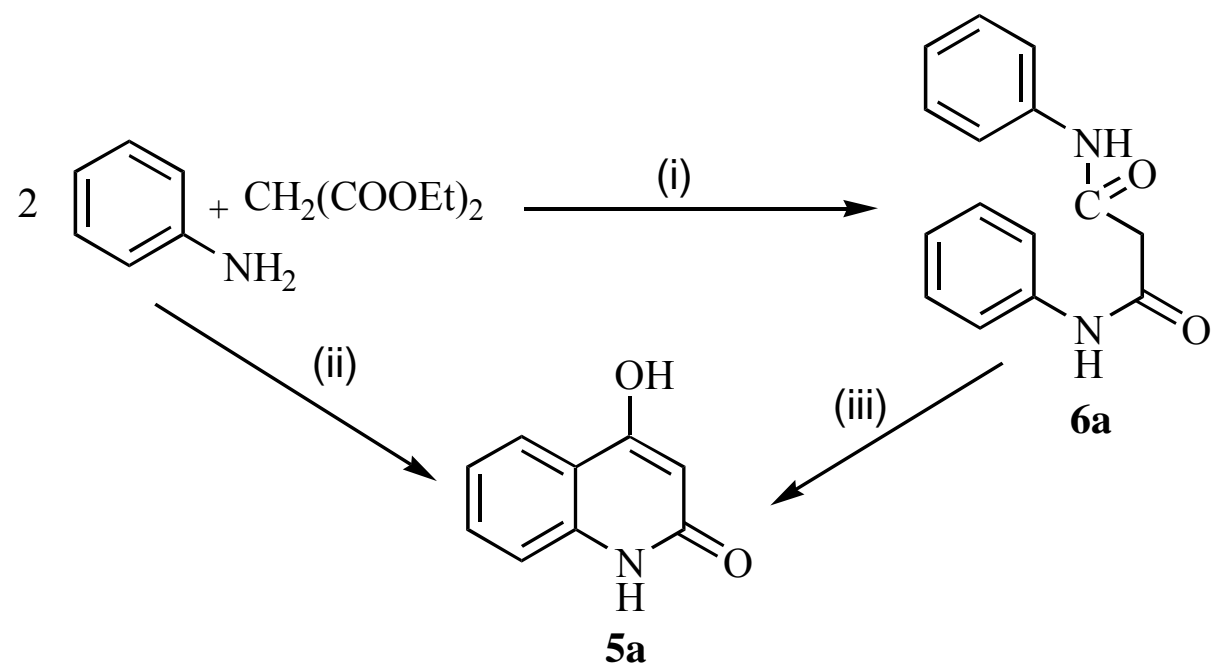

(i) neat condition, $\mathrm{mw}, 20 \mathrm{~min} \quad$ (ii) $p$-toluenesulfonic acid, $\mathrm{mw}, 6 \mathrm{~min}$ (iii) $p$-toluenesulfonic acid, $\mathrm{mw}, 3 \mathrm{~min}$

\section{Scheme 2}




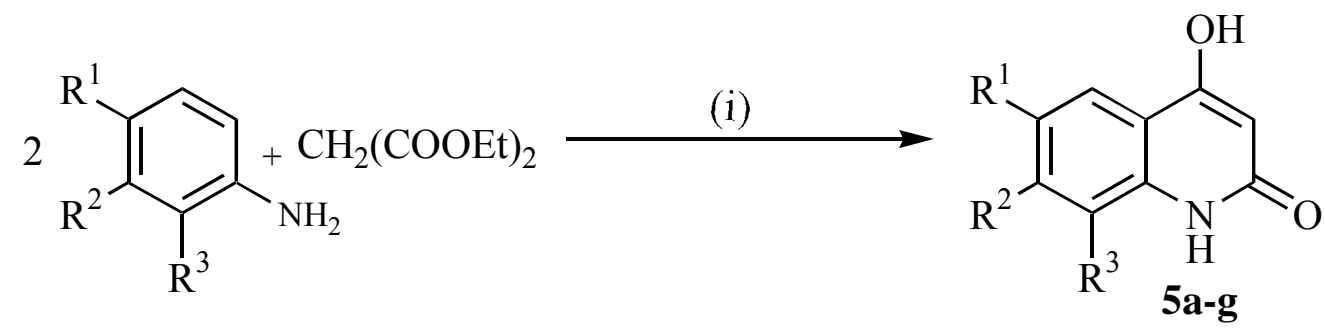

(i) p-toluenesulfonic acid, mw, 6-11 min

\section{Scheme 3}

Table 2. Microwave Synthesis of 4-Hydroxy-2-quinolinones under the catalyst $p$-toluenesulfonic acid

\begin{tabular}{cccccccc}
\hline Compound & $\mathrm{R}^{1}$ & $\mathrm{R}^{2}$ & $\mathrm{R}^{3}$ & $\begin{array}{c}\text { Reaction } \\
\text { Time }(\min )\end{array}$ & Yield $(\%)$ & $\mathrm{mp}\left({ }^{\circ} \mathrm{C}\right)$ & Lit. $\mathrm{mp}\left({ }^{\circ} \mathrm{C}\right)$ \\
\hline $\mathbf{5 a}$ & $\mathrm{H}$ & $\mathrm{H}$ & $\mathrm{H}$ & 6 & 89 & $>300$ & $>300^{23}$ \\
$\mathbf{5 b}$ & $\mathrm{H}$ & $\mathrm{H}$ & $\mathrm{OCH}_{3}$ & 6 & 90 & 248 & $245-248^{23}$ \\
$\mathbf{5 c}$ & $\mathrm{OCH}_{3}$ & $\mathrm{H}$ & $\mathrm{H}$ & 8 & 89 & 300 & $298-300^{23}$ \\
$\mathbf{5 d}$ & $\mathrm{H}$ & $\mathrm{OCH}_{3}$ & $\mathrm{OCH}_{3}$ & 10 & 85 & 246 & $244-246^{13}$ \\
$\mathbf{5 e}$ & $\mathrm{OCH}_{3}$ & $\mathrm{H}$ & $\mathrm{OCH}_{3}$ & 11 & 84 & 290 & $288-291^{16}$ \\
$\mathbf{5 f}$ & $\mathrm{H}$ & $\mathrm{H}$ & $\mathrm{CH}_{3}$ & 7 & 92 & $>300$ & $360^{18}$ \\
$\mathbf{5 g}$ & $\mathrm{CH}_{3}$ & $\mathrm{H}$ & $\mathrm{H}$ & 8 & 96 & $>300$ & $>300^{24}$ \\
\hline
\end{tabular}

Reaction of 4-hydroxy-8-methyl-2-quinolinone (5f) with dimethyl sulfate, $N, N$ dimethylformamide and potassium carbonate under microwave irradiation afforded methylated quinolinone 8 in $70 \%$ yeild in 3 min (Scheme 4). The formation of 1,8-dimethyl-4-methoxy-2quinolinone (8) is supported by the observation of peak in the expected chemical shifts of $\mathrm{OCH}_{3}$ and $\mathrm{N}-\mathrm{CH}_{3}$ protons as singlets at $\delta 3.99$ and 3.81 respectively in the ${ }^{1} \mathrm{H}-\mathrm{NMR}$ spectrum. Similar reaction of $\mathbf{5 e}$ and $\mathbf{5 g}$ with the same reagents yielded 4,6,8-trimethoxy-1-methyl-2quinolinone (7) and 1,6-dimethyl-4-methoxy-2-quinolinone (9) in good yield.

The synthesis of natural products demonstrates an interesting application of this synthetic methodology. Treatment of 4-hydroxy-2-quinolinone (5a) with $N, N$-dimethylformamide, dimethyl sulfate and potassium carbonate gave 4-methoxy-1-methyl-2-quinolinone (1) in 66\% yield in 3 min. Similarly, treatment of $5 \mathbf{b}$-d with the above reagents afforded the natural products 2, 3, and 4 in good yield. The spectroscopic properties of our synthetic materials $\mathbf{1}$ to $\mathbf{4}$ agreed well with those reported in the literature. ${ }^{10-13}$ In addition to its simple reaction conditions, this procedure has the advantage of very short reaction times, easy experimental and work-up procedures. 
<smiles>[R]c1cc2c(O)cc(=O)[nH]c2c([R])c1[R]</smiles>

5a-g<smiles>[R]c1cc2c(OC)cc(=O)n(C)c2c([R])c1[R]</smiles>

$1-4 \& 7-9$

(i) DMF, dimethyl sulfate, $\mathrm{K}_{2} \mathrm{CO}_{3}$, mw, 3-6 min

\section{Scheme 4}

\section{Conclusions}

In conclusion, we have demonstrated an efficient, mild method for the synthesis of 4-hydroxy-2quinolinones and in parallel, we have also developed an easy and fast synthetic methodology for naturally occurring 4-methoxy-1-methyl-2-quinolinone derivatives.

Table 3. Microwave Synthesis of 4-Methoxy-1-methyl-2-quinolinones

\begin{tabular}{cccccccc}
\hline Compound & $\mathrm{R}^{1}$ & $\mathrm{R}^{2}$ & $\mathrm{R}^{3}$ & $\begin{array}{c}\text { Reaction } \\
\text { Time }(\mathrm{min})\end{array}$ & Yield $(\%)$ & $\mathrm{mp}\left({ }^{\circ} \mathrm{C}\right)$ & Lit. mp $\left({ }^{\circ} \mathrm{C}\right)$ \\
\hline $\mathbf{1}$ & $\mathrm{H}$ & $\mathrm{H}$ & $\mathrm{H}$ & 3 & 66 & 100 & $100-101^{10}$ \\
$\mathbf{2}$ & $\mathrm{H}$ & $\mathrm{H}$ & $\mathrm{OCH}_{3}$ & 3 & 69 & 140 & $139-140^{11}$ \\
$\mathbf{3}$ & $\mathrm{OCH}_{3}$ & $\mathrm{H}$ & $\mathrm{H}$ & 4 & 70 & 148 & $145-146^{12}$ \\
$\mathbf{4}$ & $\mathrm{H}$ & $\mathrm{OCH}_{3}$ & $\mathrm{OCH}_{3}$ & 5 & 65 & 146 & $143-148^{13}$ \\
$\mathbf{7}$ & $\mathrm{OCH}_{3}$ & $\mathrm{H}$ & $\mathrm{OCH}_{3}$ & 6 & 67 & 163 & $161-162^{16}$ \\
$\mathbf{8}$ & $\mathrm{H}$ & $\mathrm{H}$ & $\mathrm{CH}_{3}$ & 3 & 70 & 236 & - \\
$\mathbf{9}$ & $\mathrm{CH}_{3}$ & $\mathrm{H}$ & $\mathrm{H}$ & 4 & 69 & 241 & - \\
\hline
\end{tabular}

\section{Experimental Section}

General Procedures. Melting points were recorded on Boetieus microheating table and are uncorrected. IR $(\mathrm{KBr})$ spectra were recorded on a Shimadzu-8201 FT spectrophotometer. ${ }^{1} \mathrm{H}$ NMR spectra were recorded on AMX-400 (400MHz) spectrophotometer, using TMS as an internal reference, and Mass spectra were recorded at $70 \mathrm{eV}$ on a Joel JMS-D-300 instrument. The reactions were carried out in a domestic microwave oven (KENSTAR -OM-20ESP, 2450 $\mathrm{MHz}$ ). 
General procedure for the synthesis of 4-hydroxy-2-quinolinones (5a-g). Respective aniline $(10 \mathrm{mmol})$, diethyl malonate $(5 \mathrm{mmol})$ and $p$-toluenesulfonic acid $(120 \mathrm{mg})$ were taken in a $50 \mathrm{ml}$ beaker and properly mixed with the help of a glass rod. The so-obtained paste was irradiated in a microwave oven at the power of $320 \mathrm{~W}$ for the specified time (Table 2). After irradiation, cold water was added to the reaction mixture. The solid obtained was filtered, washed with water, dried and recrystallised (Ethanol-Acetic acid) to afford the desired product in good yield.

4-Hydroxy-2-quinolinone (5a). $v_{\max }(\mathrm{KBr}) / \mathrm{cm}^{-1}$ : 3400-2900, 1660, 1606, 1514; ${ }^{1} \mathrm{H}-\mathrm{NMR}$ $\left(\mathrm{DMSO}_{\mathrm{d}}\right) \delta(\mathrm{ppm}): 5.80\left(\mathrm{~s}, 1 \mathrm{H}, \mathrm{C}_{3}-\mathrm{H}\right), 7.05-7.65(\mathrm{~m}, 3 \mathrm{H}, \mathrm{Ar}-\mathrm{H}), 7.70\left(\mathrm{~d}, 1 \mathrm{H}, \mathrm{C}_{5}-\mathrm{H}\right), 10.35(\mathrm{~s}$, $1 \mathrm{H}, \mathrm{NH}), 10.55$ (brs, 1H, OH); MS (m/z): $161\left(\mathrm{M}^{+}\right)$. Anal. Found. C 67.05; H 4.40; N 8.68. Calcd. For $\mathrm{C}_{9} \mathrm{H}_{7} \mathrm{NO}_{2} ; \mathrm{C} 67.08 ; \mathrm{H} 4.38 ; \mathrm{N} 8.69$.

4-Hydroxy-8-methoxy-2-quinolinone (5b). $v_{\max }(\mathrm{KBr}) / \mathrm{cm}^{-1}: 3400-2900,1661,1605,1514 ;{ }^{1} \mathrm{H}-$ NMR (DMSO-d $)_{6} \delta(\mathrm{ppm}): 3.90\left(\mathrm{~s}, 3 \mathrm{H}, \mathrm{C}_{8}-\mathrm{OCH}_{3}\right), 5.86\left(\mathrm{~s}, 1 \mathrm{H}, \mathrm{C}_{3}-\mathrm{H}\right), 7.14-7.40,\left(\mathrm{~m}, 2 \mathrm{H}, \mathrm{C}_{6}-\mathrm{H}\right.$ \& $\left.\mathrm{C}_{7}-\mathrm{H}\right), 7.78\left(\mathrm{~d}, 1 \mathrm{H}, \mathrm{C}_{5}-\mathrm{H}\right), 10.32(\mathrm{~s}, 1 \mathrm{H}, \mathrm{NH}), 10.54$ (brs, $\left.1 \mathrm{H}, \mathrm{OH}\right) ; \mathrm{MS}(\mathrm{m} / \mathrm{z}): 191\left(\mathrm{M}^{+}\right)$. Anal. Found. C, 62.79; H, 4.76; N, 7.30. Calcd. For $\mathrm{C}_{10} \mathrm{H}_{9} \mathrm{NO}_{3}$; C 62.82; H 4.75; N 7.33.

4-Hydroxy-6-methoxy-2-quinolinone (5c). $v_{\max }(\mathrm{KBr}) / \mathrm{cm}^{-1}: 3450-2950,1660,1606,1515 ;{ }^{1} \mathrm{H}-$ NMR (DMSO-d $)_{6} \delta(\mathrm{ppm}): 3.91\left(\mathrm{~s}, 3 \mathrm{H}, \mathrm{C}_{6}-\mathrm{OCH}_{3}\right), 5.90\left(\mathrm{~s}, 1 \mathrm{H}, \mathrm{C}_{3}-\mathrm{H}\right), 7.21-7.54,\left(2 \mathrm{~d}, 2 \mathrm{H}, \mathrm{C}_{7}-\mathrm{H}\right.$ \& $\left.\mathrm{C}_{8}-\mathrm{H}\right), 7.81\left(\mathrm{~s}, 1 \mathrm{H}, \mathrm{C}_{5}-\mathrm{H}\right), 10.34(\mathrm{~s}, 1 \mathrm{H}, \mathrm{NH}), 10.53$ (brs, $\left.1 \mathrm{H}, \mathrm{OH}\right)$; MS (m/z): $191\left(\mathrm{M}^{+}\right)$. Anal. Found. C 62.83; H 4.78; N 7.35. Calcd. For $\mathrm{C}_{10} \mathrm{H}_{9} \mathrm{NO}_{3} ; \mathrm{C} 62.82 ; \mathrm{H} 4.75 ; \mathrm{N} 7.33$.

7,8-Dimethoxy-4-hydroxy-2-quinolinone (5d). $v_{\max }(\mathrm{KBr}) / \mathrm{cm}^{-1}: 3390-2900,1660,1606,1515$; ${ }^{1} \mathrm{H}-\mathrm{NMR}\left(\mathrm{DMSO}_{\mathrm{d}}\right) \delta(\mathrm{ppm}): 3.80\left(\mathrm{~s}, 3 \mathrm{H}, \mathrm{OCH}_{3}\right), 3.92\left(\mathrm{~s}, 3 \mathrm{H}, \mathrm{OCH}_{3}\right), 6.03\left(\mathrm{~s}, 1 \mathrm{H}, \mathrm{C}_{3}-\mathrm{H}\right)$, 7.08-7.62 (2d, 2H, C $\left.5-\mathrm{H} \& \mathrm{C}_{6}-\mathrm{H}\right), 10.35$ (s, 1H, NH), 10.55 (brs, 1H, OH); MS (m/z): $221\left(\mathrm{M}^{+}\right)$. Anal. Found. C 59.75; H 5.05; N 6.30. Calcd. For $\mathrm{C}_{11} \mathrm{H}_{11} \mathrm{NO}_{4} ; \mathrm{C} 59.72 ; \mathrm{H} 5.02 ; \mathrm{N} 6.33$.

6,8-Dimethoxy-4-hydroxy-2-quinolinone (5e). $v_{\max }(\mathrm{KBr}) / \mathrm{cm}^{-1}: 3400-2900,1661,1606,1513$; ${ }^{1} \mathrm{H}-\mathrm{NMR}\left(\mathrm{DMSO}-\mathrm{d}_{6}\right) \delta(\mathrm{ppm}): 3.52\left(\mathrm{~s}, 3 \mathrm{H}, \mathrm{OCH}_{3}\right), 3.62\left(\mathrm{~s}, 3 \mathrm{H}, \mathrm{OCH}_{3}\right), 6.27\left(\mathrm{~s}, 1 \mathrm{H}, \mathrm{C}_{3}-\mathrm{H}\right), 7.58$ (s, 1H, C $7-\mathrm{H}), 7.62$ (s, 1H, C $5-\mathrm{H}), 10.33$ (s, 1H, NH), 10.54 (brs, 1H, OH); MS (m/z): $221\left(\mathrm{M}^{+}\right.$). Anal. Found. C 59.71; H 5.00; N 6.31. Calcd. For $\mathrm{C}_{11} \mathrm{H}_{11} \mathrm{NO}_{4} ; \mathrm{C} 59.72 ; \mathrm{H} 5.02 ; \mathrm{N} 6.33$.

4-Hydroxy-8-methyl-2-quinolinone (5f). $v_{\max }(\mathrm{KBr}) / \mathrm{cm}^{-1}: 3390-2900,1661,1605,1515 ;{ }^{1} \mathrm{H}-$ NMR (DMSO-d $\left.)_{6}\right) \delta(\mathrm{ppm}): 2.40\left(\mathrm{~s}, 3 \mathrm{H}, \mathrm{C}_{8}-\mathrm{CH}_{3}\right), 5.78\left(\mathrm{~s}, 1 \mathrm{H}, \mathrm{C}_{3}-\mathrm{H}\right), 7.06-7.37\left(\mathrm{~m}, 2 \mathrm{H}, \mathrm{C}_{6}-\mathrm{H} \&\right.$ $\left.\mathrm{C}_{7}-\mathrm{H}\right), 7.67$ (d, 1H, C $\left.5-\mathrm{H}\right), 10.31$ (s, 1H, NH), 10.53 (brs, 1H, OH); MS (m/z): $175\left(\mathrm{M}^{+}\right)$. Anal. Found. C 68.58; H 5.15; N 8.09. Calcd. For $\mathrm{C}_{10} \mathrm{H}_{9} \mathrm{NO}_{2}$; C 68.57; H 5.18; N 8.00.

4-Hydroxy-6-methyl-2-quinolinone (5g). $v_{\max }(\mathrm{KBr}) / \mathrm{cm}^{-1}: 3400-2900,1662,1605,1515 ;{ }^{1} \mathrm{H}-$ NMR (DMSO-d $\left.{ }_{6}\right) \delta(\mathrm{ppm}): 2.43\left(\mathrm{~s}, 3 \mathrm{H}, \mathrm{C}_{6}-\mathrm{CH}_{3}\right), 5.81\left(\mathrm{~s}, 1 \mathrm{H}, \mathrm{C}_{3}-\mathrm{H}\right), 7.10-7.40,\left(2 \mathrm{~d}, 2 \mathrm{H}, \mathrm{C}_{7}-\mathrm{H} \&\right.$ $\left.\mathrm{C}_{8}-\mathrm{H}\right), 7.68$ (s, 1H, $\left.\mathrm{C}_{5}-\mathrm{H}\right), 10.33$ (s, 1H, NH), 10.54 (brs, 1H, OH); MS (m/z): $175\left(\mathrm{M}^{+}\right)$. Anal. Found. C 68.52; H 5.17; N 8.00. Calcd. For $\mathrm{C}_{10} \mathrm{H}_{9} \mathrm{NO}_{2}$; C 68.57; H 5.18; N 8.00.

Synthesis of 4-methoxy-1-methyl-2-quinolinones (3a-g). Respective 4-hydroxy-2-quinolinone $(1 \mathrm{mmol})$ was taken in $100 \mathrm{ml}$ conical flask and to this, dimethyl sulfate $(2 \mathrm{mmol})$, potassium carbonate $(0.8 \mathrm{~g})$ and $\mathrm{N}, \mathrm{N}$-dimethyl formamide $(5 \mathrm{ml})$ were added, mixed well and irradiated for the specified time (Table 3). The reaction was monitored for every 30 seconds by the tlc. After irradiation, the reaction mixture was poured into ice water and extracted well with chloroform. The extract was dried $\left(\mathrm{Na}_{2} \mathrm{SO}_{4}\right)$ and column chromatographed to yield the pure compound. The 
spectroscopic properties of our synthetic materials $\mathbf{1}$ to $\mathbf{4}$ and $\mathbf{7}$ agreed well with those reported in the literature ${ }^{10-13}$.

1,8-Dimethyl-4-methoxy-2-quinolinone (8). $v_{\max }(\mathrm{KBr}) / \mathrm{cm}^{-1}: 1642,1580,1459 ;{ }^{1} \mathrm{H}-\mathrm{NMR}$ $\left(\mathrm{DMSO}_{-} \mathrm{d}_{6}\right) \delta(\mathrm{ppm}): 2.40\left(\mathrm{~s}, 3 \mathrm{H}, \mathrm{C}_{8}-\mathrm{CH}_{3}\right), 3.75\left(\mathrm{~s}, 3 \mathrm{H},-\mathrm{NCH}_{3}\right), 3.95\left(\mathrm{~s}, 3 \mathrm{H},-\mathrm{OCH}_{3}\right), 6.01(\mathrm{~s}$, $\left.1 \mathrm{H}, \mathrm{C}_{3}-\mathrm{H}\right), 7.06-7.68\left(\mathrm{~m}, 2 \mathrm{H}, \mathrm{C}_{6}-\mathrm{H} \& \mathrm{C}_{7}-\mathrm{H}\right), 8.01\left(\mathrm{~d}, 1 \mathrm{H}, \mathrm{C}_{5}-\mathrm{H}\right)$; $\mathrm{MS}(\mathrm{m} / \mathrm{z}): 203\left(\mathrm{M}^{+}\right)$. Anal. Found. C 70.85; H 6.46; N 6.86. Calcd. For $\mathrm{C}_{12} \mathrm{H}_{13} \mathrm{NO}_{2} ; \mathrm{C} 70.93 ; \mathrm{H} 6.45 ; \mathrm{N} 6.89$.

1,6-Dimethyl-4-methoxy-2-quinolinone (9). $v_{\max }(\mathrm{KBr}) / \mathrm{cm}^{-1}: 1643,1580,1460 ;{ }^{1} \mathrm{H}-\mathrm{NMR}$ $\left(\mathrm{DMSO}_{-} \mathrm{d}_{6}\right) \delta(\mathrm{ppm}): 2.42\left(\mathrm{~s}, 3 \mathrm{H}, \mathrm{C}_{6}-\mathrm{CH}_{3}\right), 3.81\left(\mathrm{~s}, 3 \mathrm{H},-\mathrm{NCH}_{3}\right), 3.99\left(\mathrm{~s}, 3 \mathrm{H},-\mathrm{OCH}_{3}\right), 6.02(\mathrm{~s}$, 1H, $\left.\mathrm{C}_{3}-\mathrm{H}\right), 7.20-7.62\left(2 \mathrm{~d}, 2 \mathrm{H}, \mathrm{C}_{7}-\mathrm{H} \& \mathrm{C}_{8}-\mathrm{H}\right), 7.99\left(\mathrm{~s}, 1 \mathrm{H}, \mathrm{C}_{5}-\mathrm{H}\right) ; \mathrm{MS}(\mathrm{m} / \mathrm{z}): 203\left(\mathrm{M}^{+}\right)$. Anal. Found. C 70.91; H 6.49; N 6.89. Calcd. For $\mathrm{C}_{12} \mathrm{H}_{13} \mathrm{NO}_{2} ; \mathrm{C} 70.93 ; \mathrm{H} 6.45 ; \mathrm{N} 6.89$.

\section{Acknowledgments}

The authors thank Sophisticated Instruments Facility, Indian Institute of Science, Bangalore, for recording ${ }^{1} \mathrm{H}-\mathrm{NMR}$ spectra.

\section{References}

1. Doube, D.; Blouin, M.; Brideau, C.; Chan, C.; Desmarais, C.; Ethier, D.; Falgueyret, J. P.; Friesen, R. W.; Girard, M.; Girard, Y.; Guay, J.; Tagari, P.; Young, R.N. Bioorg. Med. Chem. Lett. 1998, 8, 1255.

2. Kidwai, M.; Bhushan, K. R.; Sapra, P.; Saxena, R. K.; Gupta, R. Bioorg. Med. Chem. 2000, 8,69 .

3. Moissev, I. K.; Zemtsova, M. N.; Trakhtenberg, P. L.; Kulikowa, D. A.; Pskobkina, I.; Neshchadim, G. N.; Ostapchuk, N. V. Khim. Farm. Zh. 1998, 22, 1448.

4. Craig, J. C.; Person, P. E. J. Med. Chem. 1971, 14, 1221.

5. Narsinh, D.; Anamik, S. Ind. J. Pharm. Sci. 2001, 63, 211.

6. Dillard, R. D.; Pavey, D. E.; Benslay, D. N. J. Med. Chem. 1973, 16, 251.

7. Cobet, M.; Luckner, M. Phytochemistry 1971, 10, 1031.

8. Majumdar, K. C.; Mukhopadhyay, P. P. Synthesis 2003, 97.

9. Yong, R. L.; Hyuk, K.; Wha, S. K.; Kyung, R. M.; Youngsoo, K.; Seung, H. L. Synthesis 2001, 1851.

10. Nayar, M. N. S.; Sutar, C. V.; Bhan, M. K. Phytochemistry 1971, 10, 2843.

11. Razzakova, D. M.; Bessonova, I .A.; Yunusov, S .Yu. Khim. Prir. Soedin. 1972, 8, 133; CA 77, 72563.

12. Lamberton, J. A.; Price, J. R. Aus. J. Chem. 1953, 6, 173.

13. Storer, R.; Young, D. W.; Taylor, D. R.; Warner, J. M. Tetrahedron 1973, 29, 1721.

14. Naito, T.; Kaneko, C. Chem. Pharm. Bull. 1978, 31, 366. 
15. Coppola, G. M. J. Heterocycl. Chem. 1983, 20, 1217.

16. Storer, R.;Young, D. W. Tetrahedron 1973, 29, 1215.

17. Manandhar, M. D.; Hussaini, F. A.; Dapil, R.S.; Shob, A. Phytochemistry 1985, 24, 199.

18. Ziegler, E.; Wolf, R.; Kappe, J. Monatsche 1965, 96, 418.

19. Kabalka, G. W.; Pagni, R. M. Tetrahedron 1997, 53, 7999.

20. Roberts, B. A.; Strauss, C. R. Acc. Chem. Res. 2005, 38, 653.

21. Thamarai Selvi, S.; Nadaraj,V.; Mohan, S.; Sasi, R.; Hema, M. Bioorg. Med. Chem. In Press.

22. Lange, J. H. M.; Verveer, P. C.; Osnaburg, S. J. M.; Visser,G. M. Tetrahedron Lett. 2001, 42, 1367.

23. Shobana, N.; Yeshoda, P.; Shanmugam, P. Tetrahedron 1989, 45, 757.

24. Subramanian, M.; Mohan, P. S.; Shanmugam, P.; Rajendra Prasad, K. J. Z. Naturforsch 1992, 47b, 1016. 